\title{
Asma, rinite e atopia em escolares de duas cidades ambientalmente distintas: metrópole industrializada e região agrícola
}

\author{
Asthma, rhinitis and atopy in schoolchildren from two environmentally distinct cities: \\ industrialized metropolis and agricultural region
}

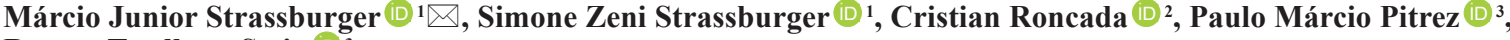 \\ Renato Tetelbom Stein $\left(\mathbb{D}^{3}\right.$ \\ 1 Universidade Regional do Noroeste do Fstado do Rio Grande do Sul, Departamento de Ciências da Vida, Curso de Fisioterapia. ljuí, RS, Brasil. \\ 2 Centro Universitário da Serra Gaúcha, Faculdade de Educação Física. Caxias do Sul, RS, Brasil. \\ 3 Pontifícia Universidade Católica do Rio Grande do Sul, Faculdade de Medicina. Porto Alegre, RS, Brasil.
}

Como citar este artigo (How to cite this article):

Strassburger MJ, Strassburger SZ, Roncada C, Pitrez PM, Stein RT. Asma, rinite e atopia em escolares de duas cidades ambientalmente distintas: metrópole industrializada e região agrícola, (Asthma, rhinitis and atopy in schoolchildren from two environmentally distinct cities: industrialized metropolis and agricultural region). Sci Med. 29(4):e34336. https://doi.org/10.15448/1980-6108.2019.4.34336

\section{RESUMO}

OBJETIVO: Analisar as características da asma entre escolares de região metropolitana e região agrícola.

MÉTODOS: Os escolares de ambos locais, responderam questionário de diagnóstico epidemiológico da asma e características clínicas da asma, rinite e atopia conforme ISAAC. Também foram avaliados pelo Teste de Controle da Asma e medida de atividade física.

RESULTADOS: Entre os 290 e 123 asmáticos avaliados na região metropolitana e região agrícola, respectivamente, a prevalência de asma foi $20,4 \%$ e $19,2 \%$. Os asmáticos da região agrícola apresentaram maior frequência de rinite $(87 \% v s .48,8 \%$, p $<0,001)$ e atopia $(81,4 \% v s$. $63,5 \%, p=0,005)$ Não foi observada diferença em medidas de controle da doença $(51,9 \%$ vs. 47,2\%, $\mathrm{p}=0,378)$. A combinação asma com rinite ou atopia apresentou risco significativo para o controle da doença nos escolares da região agrícola (OR: 2,300; IC95\%:1,867-2,833).

CONCLUSÃO: Não houve diferença na frequência de asma e controle da doença entre os escolares estudados. Contudo, os escolares da região agrícola apresentam maiores taxas de rinite e atopia o que interferiu negativamente no controle da asma. Estes achados sugerem que asma em populações com meio ambientes distintos têm características clínicas distintas, que devem ser levadas em conta na definição de estratégias de prevenção e manejo clínico.

DESCRITORES: Asma, prevalência, criança, rinite, alergia.

\section{ABSTRACT}

AIM: To analyze the characteristics of asthma among students from metropolitan and agricultural regions.

METHODS: Students from both locations answered a questionnaire for epidemiological diagnosis of asthma and clinical characteristics of asthma, rhinitis and atopy according to ISAAC. They were also evaluated by the Asthma Control Test and measure of physical activity.

RESULTS: Among the 290 and 123 asthmatics evaluated in the metropolitan region and agricultural region, respectively, the prevalence of asthma was $20.4 \%$ and $19.2 \%$. Asthmatics in the agricultural region had a higher frequency of rhinitis $(87 \% v s .48 .8 \%, \mathrm{p}<0.001)$ and atopy ( $81.4 \%$ vs. $63.5 \%, \mathrm{p}=0.005)$ No difference was observed in disease control measures $(51.9 \%$ vs. $47.2 \%, \mathrm{p}=0.378)$. The combination asthma with Rhinitis or atopy presented significant risk for disease control in schoolchildren in the agricultural region (OR: 2,300; 95\% CI: 1,867-2,833). CONCLUSION: There was no difference in asthma frequency and disease control among the students studied. However, the students of agricultural region have higher rates of rhinitis and atopy which negatively interfered with asthma control. These findings suggest that Asthma in populations with different environments have different clinical characteristics, which should be taken into account when defining prevention strategies and clinical management.

KEYWORDS: Asthma, prevalence, child, rhinitis, allergy. 
Abreviaturas: ISAAC, International Study of Asthma and Allergies in Childhood; ACT, Teste de Controle de Asma.

\section{INTRODUÇÃO}

Asma é uma doença heterogênea, caracterizada por hiperresponsividade brônquica e inflamação crônica das vias aéreas [1]. É a condição crônica mais comum da infância [2] e apresenta prevalência variável entre os diferentes países e distintas cidades do mesmo país [3].

Na América Latina a prevalência média atual de sintomas de asma em adolescentes, verificada pela Fase III do International Study of Asthma and Allergies in Childhood (ISAAC) é de $17 \%$, com alta variação entre os países $(6,9 \%-37,6 \%)$ [4]. No Brasil, as taxas de prevalência de asma ativa em adolescentes entre $11,8 \%$ e $30,5 \%[5,6]$.

A asma tem características multicausais e sua manifestação apresenta relação com as condições ambientais, geográficas, socioeconômicas, emocionais e genéticas. Além disso, estudos descrevem diferenças para manifestação e intensidade dos sintomas de asma entre regiões urbanas e rurais [7-11].

As populações urbanas de regiões metropolitanas estão susceptíveis às condições ambientais relacionadas a urbanização, industrialização e ao intenso tráfego rodoviário. Enquanto que as populações de pequenas e medias cidades, cuja principal atividade econômica é a agricultura, caracterizadas como ambientes semirrurais, estão expostas a poluição ambiental oriunda do manejo dos diferentes cultivos agrícolas e submetidas a intensa invasão aérea de pólens e poeira resultantes da atividade agrícola $[9,11]$.

Neste contexto, objetivamos analisar as características da asma entre populações pediátricas de locais com características ambientais distintas, escolares de região metropolitana e cidade de médio porte em região agrícola no sul do Brasil.

\section{MÉTODOS}

O presente estudo analisa os dados obtidos em duas cidades do sul do Brasil, entre escolares da rede pública de ensino, com idade de 8 a 16 anos. Foram obtidos dados de uma metrópole industrializada (Porto Alegre) e de uma cidade de porte médio em região agrícola (Ijuí, município localizado no noroeste do estado do RS). Em Porto Alegre foram avaliados 2.500 escolares, enquanto que em Ijuí-RS foram incluídos 1.688 escolares. As aprovações do Comitês de Ética em Pesquisa da PUCRS têm numeração de pareceres consubstanciados 73583/2012 e $131568 / 2012$.

A coleta dos dados foi organizada a partir de duas etapas distintas. A primeira etapa consistiu na classificação socioeconômica, a partir do Critério de Classificação Econômica Brasil [12], e triagem dos escolares com asma. Para a triagem dos escolares com asma foi utilizado o instrumento para o diagnóstico epidemiológico de asma de acordo com o protocolo ISAAC [13]. O instrumento é composto por quatro questões que investigam diagnóstico médico alguma vez na vida, crises de asma nos últimos 12 meses e em algum momento na vida, além da utilização de medicamentos para asma nos últimos 12 meses. Para que os escolares fossem caracterizados como asmáticos, as quatro perguntas deveriam ter respostas positivas.

$\mathrm{Na}$ segunda etapa das pesquisas, os escolares classificados com asma, a partir da triagem da primeira etapa, receberam vista domiciliar para responder aos questionários e realizar testes específicos. Para investigação dos sintomas respiratórios e características clínicas da asma, rinite e atopia, foi utilizado um questionário validado para o Brasil, que é usado pelo estudo ISAAC [14]. O controle da doença foi avaliado através do Teste de Controle de Asma (ACT) [15], com ponto de corte em $\geq 20$ pontos para categorização da asma controlada. O nível de atividade física foi investigado através de um protocolo específico [16], que contabilizou a quantidade de tempo semanal em atividades físicas realizadas. Foram classificados como escolares sedentários aqueles com menos de 300 minutos semanais acumulados de atividade física relatada.

Para fins de análise estatística, as variáveis contínuas são apresentadas por média e desvio padrão ou mediana e intervalo interquartil, conforme a simetria das variáveis. As variáveis categóricas estão apresentadas por frequências absolutas e relativas. Para comparação das variáveis, com distribuição normal entre grupos foi aplicado o Teste t de Student, e o teste de Mann Whitney para as variáveis com distribuição anormal. As relações entre as variáveis categóricas foram testadas pelo método de quiquadrado e a razão de chances entre as variáveis foi testado pelo método de Odds Ratio. As diferenças entre grupos ou análises de desfecho foram consideradas significativamente diferentes com $p<0,05$. Todas as análises foram realizadas usando IBM SPSS Statistics 18. 


\section{RESULTADOS}

$\mathrm{Na}$ primeira etapa dos estudos, foram coletados dados de 2.500 escolares residentes em metrópole industrializada (Porto Alegre) e 1.691 escolares de uma cidade localizada em região agrícola (Ijuí), para identificação da prevalência de asma. Segundo critérios do (ISAAC), na metrópole o número de escolares com asma foi de $511(20,4 \%)$, enquanto que na região agrícola foi de $324(19,2 \%)$.

Conforme a Tabela 1, identificamos que os escolares com asma residentes em Ijuí são majoritariamente pertencentes de classes socioeconômicas mais altas $(52,8 \%)$, apresentando maior prevalência de rinite $(87 \%)$, atopia $(81,4 \%)$ e maior tempo de atividade física semanal $(\mathrm{p}=0,033)$. Já os escolares com asma de Porto Alegre apresentaram maior frequência de exposição ao tabagismo em ambiente residencial $(55,5 \%)$.

Além das diferenças observadas nas frequências isoladas de rinite e atopia, quando combinamos a ocorrência de asma com alguma comorbidade (rinite ou atopia), percebe-se que a maioria dos escolares estudados apresenta asma associada com pelo menos uma comorbidade [Porto Alegre, 107 (84,3\%); Ijuí, $115(93,5 \%) ; p=0,021]$, sendo essa situação mais frequente em Ijuí.

De forma geral, entre os escolares estudados, não houve diferença estatística para frequência do controle da doença $(p=0,378)$ e nos escores do ACT $(p=0,617)$. Porém, quando comparamos escolares com asma e mais uma condição associada (rinite ou atopia) com escolares com asma sem outra condição, identificamos que os escolares de Ijuí com a associação de asma e rinite ou atopia apresentam menor pontuação no ACT que seus colegas com apenas asma $(18,74 \pm 3,81$ vs. $22,00 \pm 1,51 ; \mathrm{p}=0,018)$, ou seja essa associação contribui para menor controle da doença. Os escolares de Porto Alegre não apresentaram diferença para essa comparação $(19,41 \pm 3,85$ vs. $18,95 \pm 5,12 ; \mathrm{p}=0,643)$.

Na Tabela 2 apresentamos o risco de cada variável interferir no controle da asma. Apesar de haver importante variação nas frequências das comorbidades nos locais da pesquisa, apenas a condição da asma associada com rinite ou atopia apresentou risco para o controle da doença nos escolares de Ijuí (OR: 2,300; IC95\%:1,867-2,833). Para os escolares de Porto Alegre não houve essa relação estatística.

Tabela 1. Caracterização dos escolares com asma de acordo com o local de estudo.

\begin{tabular}{|c|c|c|c|}
\hline & Porto Alegre & ljuí & $\mathbf{P}$ \\
\hline Idade, em anos $(M \pm D P)$ & $10,79 \pm 2,24$ & $11,35 \pm 1,97$ & $0,011^{*}$ \\
\hline Sexo feminino $[N / D(\%)]$ & $150 / 290(51,7)$ & $63 / 123(51,2)$ & $0,925^{\times}$ \\
\hline Classificação Socioeconômica A+B [N/D(\%)] & $53 / 290(18,3)$ & $65 / 123(52,8)$ & $<0,001^{x}$ \\
\hline Asma + Rinite [N/D(\%)] & $70 / 119(58,8)$ & $107 / 123(87,0)$ & $<0,001^{x}$ \\
\hline Asma + Atopia $[\mathrm{N} / \mathrm{D}(\%)]$ & $80 / 126(63,5)$ & $70 / 86(81,4)$ & $0,005^{\times}$ \\
\hline Asma + Rinite ou Atopia [N/D(\%)] & $107 / 127(84,3)$ & $115 / 123(93,5)$ & $0,021^{x}$ \\
\hline Asma controlada $[\mathrm{N} / \mathrm{D}(\%)]$ & $151 / 290(51,9)$ & $58 / 123(47,2)$ & $0,378^{x}$ \\
\hline Escore ACT $(\mathrm{M} \pm \mathrm{DP})$ & $19,16 \pm 3,96$ & $18,95 \pm 3,78$ & $0,617^{*}$ \\
\hline Tabagismo residencial $[\mathrm{N} / \mathrm{D}(\%)]$ & $66 / 119(55,5)$ & $31 / 94(33,0)$ & $0,001^{x}$ \\
\hline Tempo de atividade física semanal (M-IQ) & $160(50-382,5)$ & $250(100-400)$ & $0,033^{\sharp}$ \\
\hline
\end{tabular}

N/D(\%): Número de casos, denominador e frequência relativa (adota-se como denominador o número de avaliações respondidas para cada variável): M DDP: Média e desvio padrão; M-IQ: Mediana e Intervalo Interquartil em minutos por semana; ACT: Teste de Controle da Asma.

* Teste t de Student; ${ }^{\times}$Teste Quiquadrado; ${ }^{\text {T Teste de Mann-Whitney }}$

Tabela 2. Relação das variáveis investigadas com o risco para a falta de controle da asma nos escolares com asma de Porto Alegre e ljuí, RS.

\begin{tabular}{lcc}
\hline & Porto Alegre & Ijuí \\
Asma e Rinite & OR (IC95\%) & OR (IC95\%) \\
Asma e Atopia & $1,190(0,786-1,801)$ & $1,470(0,764-2,831)$ \\
Asma, Rinite ou Atopia & $1,273(0,831-1,951)$ & $1,273(0,703-2,306)$ \\
Sedentarismo & $1,003(0,592-1,701)$ & $2,300(1,867-2,833)^{*}$ \\
Tabagismo residencial & $1,262(0,957-1,663)$ & $1,210(0,850-1,723)$ \\
Classe Socioeconômica & $1,262(0,837-1,903)$ & $1,016(0,686-1,505)$ \\
Sexo masculino & $1,072(0,814-1,411)$ & $1,358(0,920-2004)$ \\
\hline
\end{tabular}

OR: Odds Ratio; IC95\%: Intervalo de Confiança de 95\%; * p-valor <0,05. 
Além das alergias, também encontramos diferenças significativas para o tempo de atividade física semanal. Os escolares de Ijuí apresentam maior tempo semanal gasto em atividades físicas que os residentes de Porto Alegre [250 (100-400) vs. 160 (50-382,5); $\mathrm{p}=0,033$ ]. Porém, quando realizamos a categorização dos escolares em ativos ou sedentários, não há diferença significativa entre as frequências observadas [Porto Alegre, 195 (67,2\%) vs. Ijuí, 52 (58,5\%); $\mathrm{p}=0,091]$. Contudo, conforme a tabela 2 , o nível de atividade física não interferiu no controle da asma dos escolares de Porto Alegre e Ijuí.

A distribuição da classificação socioeconômica, também distinta entre os locais de pesquisa, não apresentou risco significativo sobre o controle da doença (Tabela 2). Apesar disso, quando comparamos os escores do ACT entre os escolares das classes $\mathrm{A}+\mathrm{B}$ e classes $\mathrm{C}+\mathrm{D}+\mathrm{E}$, em cada local de pesquisa, identificamos que em Ijuí, os escolares das classes superiores apresentam maiores valores para o teste de controle de asma $(19,71 \pm 3,36$ vs. $18,10 \pm 4,08 ; \mathrm{p}=0,018)$, porém dentro da classificação de não controlados. Para os escolares de Porto Alegre essa diferença não é significativa $(19,62 \pm 3,88 v s .19,06 \pm 3,98 ; \mathrm{p}=0,350)$.

A Tabela 3 apresenta os aspectos relacionados ao impacto do tratamento e controle da asma. Apesar da diferença significativa, ambos os locais apresentam baixa frequência de acompanhamento clínico para os escolares com asma. A existência de plano de tratamento prescrito e uso contínuo de medicamento também é baixo. Apenas para uso contínuo de medicação pelos escolares de Ijuí há diferença nos escores do teste de controle da asma, sendo que os escolares com uso contínuo de medicação apresentam menores valores para o ACT $(17,39 \pm 3,35$ vs. 19,88 $\pm 3,74 ; \mathrm{p}<0,001)$. Para os escolares de Porto Alegre essa relação não ocorreu $(18,89 \pm 4,02$ vs. 19,42 $\pm 4,10 ; \mathrm{p}=0,524)$.

Importante destacar que, embora os escolares de Ijuí apresentem maior frequência de acompanhamento clínico, não houve diferença estatística para os valores de visita a emergência, internação hospitalar e absenteísmo escolar.

\section{DISCUSSÃO}

Este estudo demonstrou elevada prevalência de asma e baixo nível de controle da doença em escolares de regiões com características ambientais distintas (metrópole industrializada e cidade de região agrícola). Contudo, a prevalência de rinite e atopia foi significativamente maior nos escolares residentes da região agrícola (Ijuí), o que interferiu negativamente no controle da doença comparado aos escolares da região metropolitana (Porto Alegre).

O Brasil é um dos países da América Latina com maior número de centros de pesquisa vinculados ao ISAAC, com 58.418 adolescentes avaliados e média nacional de asma atual de 18,7\% [4]. Além disso, uma revisão sistemática, que reuniu dados referente a prevalência da asma nos centros brasileiros oficiais do ISAAC e outras de pesquisas que usaram a mesma metodologia, demonstrou prevalência asma de 18,2\% em Porto Alegre [5]. Ambos os locais avaliados por este estudo, mostraram frequência de asma próxima às descritas (Porto Alegre $20,4 \%$ vs. 19,2\% em Ijuí), porém chama a atenção que escolares residentes em Ijuí, área menos populosa, pouco industrializada e baixo fluxo rodoviário, apresente os mesmos índices de regiões metropolitanas.

Também não houve diferença significativa na frequência de asma controlada entre Porto Alegre e Ijuí (51,9\% vs. 47,2\%, $\mathrm{p}=0,378)$, apesar dos escolares de Ijuí relatarem maior frequência de acompanhamento clínico realizado. A falta de controle da doença é um problema global, sendo que estudos realizados na América Latina indicam que $36 \%$ a $40 \%$ dos escolares não atingem bom controle da doença $[4,17]$.

Tabela 3. Descrição dos resultados referente ao impacto do tratamento e controle da asma

\begin{tabular}{|c|c|c|c|}
\hline & Porto Alegre & ljuí & \multirow{2}{*}{$\mathbf{p}$} \\
\hline & $N(\%)$ & $\mathbf{N}(\%)$ & \\
\hline Tem plano de tratamento prescrito & $41(36,9)$ & $59(49,2)$ & 0,061 \\
\hline Faz uso contínuo de medicação & $38(34,5)$ & $46(38,3)$ & 0,551 \\
\hline Faz acompanhamento clínico & $29(25,7)$ & $48(39,3)$ & $0,026^{*}$ \\
\hline Alguma visita à Emergência nos últimos 12 meses & $16(13,9)$ & $18(15,0)$ & 0,686 \\
\hline Alguma internação hospitalar nos últimos 12 meses & $9(8,5)$ & $10(8,3)$ & 0,475 \\
\hline Algum dia de aula perdido nos últimos 12 meses & $68(61,3)$ & $79(64,8)$ & 0,357 \\
\hline
\end{tabular}

$N(\%)$ : Frequências absolutas e relativas; * Teste Quiquadrado 
O controle da asma pode sofrer interferência pela presença de diferentes comorbidades, sendo a rinite e atopia as mais comuns. A rinite é a comorbidade com maior frequência entre os asmáticos (aproximadamente $78 \%$ ) $[18,19]$. Estudo realizado sobre a sensibilização a aeroalergenos em adultos jovens em Passo Fundo (distante $168 \mathrm{~km}$ de Ijuí e $289 \mathrm{~km}$ de Porto Alegre) encontrou frequência de rinite de $72,5 \%$, e dentre eles, $88 \%$ apresentou prick-test positivo para ácaros e pólens [20].

Em Ijuí a rinite é mais elevada que a descrita, está presente em $87 \%$ dos escolares com asma, enquanto que em Porto Alegre está abaixo da relatada pela literatura, acorrendo em 58,8\% dos casos. As frequências encontradas para atopia estão elevadas em ambos os locais de estudo, em Ijuí ocorre em 81,4\% dos escolares com asma, e em Porto Alegre afeta $63,5 \%$, enquanto que estudos relatam taxas de atopia entre $42,6 \%$ e $50,4 \%[21,22]$.

Provavelmente dois fatores poderiam explicar estas diferenças. Uma é que em Ijuí os escolares são de nível socioeconômico mais elevado $(52,8 \%$ vs. 18,3\% - Porto Alegre) e há literatura consistente que indica que quanto melhor é essa situação, maior o índice de doenças alérgicas $[10,23]$. Outro aspecto importante são as características regionais, sendo Ijuí uma região agrícola, é exposta a contaminação ambiental resultante do manejo de diferentes plantações, o que pode elevar a prevalência de sintomas de doenças alérgicas. Segundo informações do Instituto Brasileiro de Geografia e Estatística (sidra.ibge.gov. br/pesquisa/pam/tabelas) a produção agrícola de Ijuí está relacionada, principalmente com a aveia, milho, soja e trigo, atingindo 57.191 hectares de área cultivada.

Paralelo às altas prevalências de asma, rinite $\mathrm{e}$ atopia, chama atenção em nossos resultados a elevada frequência de concomitância de asma associada com rinite ou atopia. Em Porto Alegre essa associação ocorre em $84,3 \%$ dos casos, e é maior ainda em Ijuí, 93,5\%. Enquanto isso, a literatura descreve essa situação em apenas 22,6\% dos asmáticos [22].

Para os escolares de Ijuí, a associação da asma com alguma outra condição (rinite ou atopia), apresentou risco para o controle da doença. Ou seja, a presença de rinite ou atopia associada a asma proporcionou menor controle da doença para os escolares residentes da região agrícola. Estudos mostram que a falta de controle de comorbidades, como rinite e atopia, podem interferir no controle da asma [18,24], sendo que as características estão diretamente relacionadas com o controle das doenças alérgicas.
Diferente das doenças alérgicas, o efeito da atividade física sobre a asma não é claro. Alguns estudos indicam que o mal controle da doença está associada com a redução dos níveis de atividades física, enquanto que outros afirmam que altos índices de atividades física estão associados com baixo controle da asma [25-27]. Em nosso estudo, apesar da maior quantidade de atividade física realizada pelos escolares de Ijuí, identificamos alta frequência de sedentarismo entre os escolares de ambos locais de pesquisa, porém essa condição não interferiu no controle da asma.

Com a diversidade de variáveis que podem interferem no manejo asma, o acompanhamento clínico e a adesão ao tratamento são determinantes para o controle da doença e suas comorbidades, contudo a literatura mostra que a adesão ao tratamento é baixa entre os asmáticos [28]. Em nosso estudo, menos da metade dos escolares têm plano de tratamento prescrito e uso contínuo de medicamento, e apenas 39,3\% dos escolares de Ijuí realizam acompanhamento clínico e $25,7 \%$ dos escolares de Porto Alegre $(p=0,026)$.

Apesar das diferenças encontradas para a frequência de acompanhamento clínico, prevalência de rinite e atopia, características regionais e condição socioeconômica, não houve diferença no impacto da doença para os escolares estudados, quanto a visita à emergência, hospitalizações e absenteísmo escolar (apesar de estar elevado em ambos os locais).

A asma tem altos índices de prevalência nas duas cidades estudadas no sul do Brasil, mesmo com perfis ambientais e socioeconômicos diferentes. Rinite e atopia, embora elevados em ambos locais de estudo, foram significativamente mais frequentes entre os asmáticos da região agrícola (Ijuí). Da mesma forma, os escolares de Ijuí têm maior interferência no controle da doença mediante a presença de comorbidades. Estes achados sugerem que asma em populações com meio ambientes distintos têm características específicas e que devem ser levadas em conta na definição de estratégias de prevenção e manejo clínico.

\section{NOTAS}

Parte deste trabalho é resultado de tese de doutorado de um dos autores (MJS), intitulada "Caracterização da asma entre populações pediátricas distintas: metrópole industrializada e cidade de porte médio do Sul do Brasil".

Apoio financeiro

Este estudo não recebeu apoio financeiro de fontes externas.

Declaração de conflito de interesses

Os autores declaram não haver conflitos de interesses relevantes ao conteúdo deste estudo. 


\section{Contribuições dos autores}

Todos os autores fizeram contribuições substanciais para concepção, ou delineamento, ou aquisição, ou análise ou interpretação de dados; e redação do trabalho ou revisão crítica; e aprovação final da versão para publicação.
Disponibilidade dos dados e responsabilidade pelos resultados

Todos os autores declaram ter tido total acesso aos dados obtidos e assumem completa responsabilidade pela integridade destes resultados.

\section{REFERÊNCIAS}

1. GINA. Global Strategy for Asthma Management and Prevention. Fontana: GINA; 2017.

2. Castro-Rodriguez JA. The Asthma Predictive Index: a very useful tool for predicting asthma in young children. J Allergy Clin Immunol. 2010;126(2):212-6. https://doi.org/10.1016/j.jaci.2010.06.032

3. Pearce N, Aït-Khaled N, Beasley R, Mallol J, Keil U, Mitchell E, Robertson C; ISAAC Phase Three Study Group. Worldwide trends in the prevalence of asthma symptoms: phase III of the International Study of Asthma and Allergies in Childhood (ISAAC). Thorax. 2007;62(9):758-66. https://doi.org/10.1136/thx.2006.070169

4. Solé D, Aranda CS, Wandalsen GF. Asthma: epidemiology of disease control in Latin America-short review. Asthma Res Pract. 2017;3(1):4. https://doi.org/10.1186/s40733-017-0032-3

5. Solé D, Camelo-Nunes IC, Wandalsen GF, Mallozi MC. Asthma in children and adolescents in Brazil: contribution of the International Study of Asthma and Allergies in Childhood (ISAAC). Rev Paul Pediat. 2014;32(1):114-25. https://doi.org/10.1590/s0103-05822014000100018

6. Roncada C, de Oliveira SG, Cidade SF, Sarria EE, Mattiello R, Ojeda BS, Dos Santos BR, Gustavo Ada S, Pinto LA, Jones MH1, Stein RT, Pitrez PM. Burden of asthma among inner-city children from Southern Brazil. J Asthma. 2016;53(5):498-504. https://doi.org/10.3109/02770903.2015.1108438

7. Ocampo J, Gaviria R, Sánchez J. Prevalence of asthma in Latin America. Critical look at ISAAC and other studies. Rev Alerg Mex (Tecamachalco, Puebla, Mexico: 1993). 2017;64(2):188-97.

8. de Valois Correia Junior MA, Sávio Cavalcanti Sarinho E, Rizzo JA, Wanick Sarinho S. Menor prevalência e maior gravidade da asma em clima quente e seco. J Pediatr. 2017;93(2).

9. Schröder PC, Li J, Wong GW, Schaub B. The rural-urban enigma of allergy: What can we learn from studies around the world? Pediatr Allergy Immunol. 2015;26(2):95-102. https://doi.org/10.1111/pai.12341

10. Kliś K, Żurawiecka M, Suder A, Teul I, Borowska-Strugińska B, Suliga E, Wronka I. Influence of Socioeconomic Factors on Self-Reported Prevalence of Allergic Diseases Among Female University Students. Adv Exp Med Biol. 2017;1020:17-24. https://doi.org/10.1007/5584_2017_14

11. Rodriguez A, Vaca MG, Chico ME, Rodrigues LC, Barreto ML, Cooper PJ. Rural to urban migration is associated with increased prevalence of childhood wheeze in a Latin-American city. BMJ Open Respir Res. 2017;4(1):e000205. https://doi.org/10.1136/bmjresp-2017-000205

12. Brasil CdC. Critério de classificação econômica Brasil: Associação Brasileira de Empresas de Pesquisa (ABEP); 2008.

13. Committee IS. Worldwide variations in the prevalence of asthma symptoms: ISAAC. Eur Respir J. 1998;12:315-35.

14. Solé D, Yamada E, Vana AT, Werneck G, Solano de Freitas L, Sologuren MJ, Brito M, Rosário Filho NA, Stein RT, Mallol J. International Study of Asthma and Allergies in Childhood (ISAAC): prevalence of asthma and asthmarelated symptoms among Brazilian schoolchildren. J Investig Allergol Clin Immunol. 2001;11(2):123-8. https://doi. org/10.1157/13057763

15. Roxo JPF, Ponte EV, Ramos DCB, Pimentel L, D’Oliveira Junior A, Cruz Filho ÁASd. Validação do Teste de Controle da Asma em português para uso no Brasil. J. bras. pneumol. 2010 Apr;36(2):159-66, https://doi.org/ 10.1590/s1806-37132010000200002

16. Hallal PC, Bertoldi AD, Gonçalves H, Victora CG. [Prevalence of sedentary lifestyle and associated factors in adolescents 10 to 12 years of age]. Cad Saude Publica. 2006;22(6):1277-87. https://doi.org/10.1590/s0102$311 \times 2006000600017$ 
17. Maspero JF, Jardim JR, Aranda A, Tassinari P, Gonzalez-Diaz SN, Sansores RH, Moreno-Cantu JJ, Fish JE. Insights, attitudes, and perceptions about asthma and its treatment: findings from a multinational survey of patients from Latin America. World Allergy Organ J. 2013 Nov 4;6(1):19. https://doi.org/10.1186/1939-4551-6-19

18. Brożek JL, Bousquet J, Agache I, Agarwal A, Bachert C, Bosnic-Anticevich S, Brignardello-Petersen R, Canonica GW, Casale T, Chavannes NH, Correia de Sousa J, Cruz AA, Cuello-Garcia CA, Demoly P, Dykewicz M, Etxeandia-Ikobaltzeta I, Florez ID, Fokkens W, Fonseca J, Hellings PW, Klimek L, Kowalski S, Kuna P, Laisaar KT, Larenas-Linnemann DE, Lødrup Carlsen KC, Manning PJ, Meltzer E, Mullol J, Muraro A, O’Hehir R, Ohta K, Panzner P, Papadopoulos N, Park HS, Passalacqua G, Pawankar R, Price D, Riva JJ, Roldán Y, Ryan D, Sadeghirad B, Samolinski B, Schmid-Grendelmeier P, Sheikh A, Togias A, Valero A, Valiulis A, Valovirta E, Ventresca M, Wallace D, Waserman S, Wickman M, Wiercioch W, Yepes-Nuñez JJ, Zhang L, Zhang Y, Zidarn M, Zuberbier T, Schünemann HJ. J Allergy Clin Immunol. 2017;140(4):950-8. https://doi.org/10.1016/j.jaci.2017.03.050

19. Camelo-Nunes IC, Solé D. Rinite alérgica: indicadores de qualidade de vida. J Bras Pneumol. 2010;36(1):124-33. https://doi.org/10.1590/s1806-37132010000100017

20. Dal Bello MS, Schneider ML, Doring M, Gomes LM, Mistura TC. Sensibilização a aeroalérgenos em adultos jovens vivendo na região sul do Brasil. Braz J Allergy Immunol. 2013;1(4):223-8.

21. Zdraveska M, Dimitrievska D, Todevski D, Gjorcev A, Janeva E, Pavlovska I, Zafirova-Ivanovska B2. Joint incidence of asthma and rhinitis in macedonia. Open Respir Med J. 2015;9:52-8. https://doi.org/10.2174/1874306401 509010052

22. Soto-Martínez ME, Yock-Corrales A, Camacho-Badilla K, Abdallah S, Duggan N, Avila-Benedictis L, et al. The current prevalence of asthma, allergic rhinitis, and eczema related symptoms in school-aged children in Costa Rica. J Asthma. 2018:1-9. https://doi.org/10.1080/02770903.2018.1455860

23. Petry C, Pereira MU, Pitrez PM, Jones MH, Stein RT. The prevalence of symptoms of sleep-disordered breathing in Brazilian schoolchildren. J Pediatr (Rio J). 2008;84(2):123-9. https://doi.org/10.2223/jped.1770

24. Montoro J, Del Cuvillo A, Mullol J, Molina X, Bartra J, Dávila I, Ferrer M, Jáuregui I, Sastre J, Valero A. Validation of the modified allergic rhinitis and its impact on asthma (ARIA) severity classification in allergic rhinitis children: the PEDRIAL study. Allergy. 2012;67(11):1437-42. https://doi.org/10.1111/all.12011

25. Lövström L, Emtner M, Alving K, Nordvall L, Borres MP, Janson C, Malinovschi A. High levels of physical activity are associated with poorer asthma control in young females but not in males. Respirol. 2016;21(1):79-87. https://doi. org/10.1111/resp. 12671

26. Eijkemans M, Mommers M, Draaisma JM, Thijs C, Prins MH. Physical activity and asthma: a systematic review and meta-analysis. PLoS One. 2012;7(12):e50775. https://doi.org/10.1371/journal.pone.0050775

27. Carson KV, Chandratilleke MG, Picot J, Brinn MP, Esterman AJ, Smith BJ. Physical training for asthma. Cochrane Database Syst Rev. 2013(9):CD001116. https://doi.org/10.1002/14651858.cd001116.pub4

28. Neffen H, Fritscher C, Cuevas Schacht F, Levy G, Chiarella P, Soriano JB, Mechali D; AIRLA Survey Group. Asthma control in Latin America: the asthma insights and reality in Latin America (AIRLA) survey. Rev Panam Salud Publica 2005;17(3):191-7. https://doi.org/10.1590/s1020-49892005000300007 